\title{
Intervenciones para reducir el embarazo no buscado en adolescentes
}

Interventions to reduce unintended pregnancies among adolescents: systematic review of randomized controlled trials. DiCenso A, Guyatt G, William A, Griffith L. BMJ 2002;324:1426-34.

\section{Objetivo}

Evaluar la efectividad de estrategias de prevención dirigidas a retrasar el inicio de las relaciones sexuales, mejorar el uso de la planificación del embarazo y reducir la incidencia de embarazos no deseados en adolescentes.

\section{Fuente y Selección de Datos}

Estudios aleatorizados, publicados y no publicados que hubieran evaluado programas de prevención del embarazo en adolescentes (entre 11 y 18 años) realizados en escuelas, comunidades y clínicas de planificación familiar con el objetivo de lograr un retraso en el inicio de las relaciones sexuales, uso efectivo de control de la natalidad o prevención de embarazos no deseados. Se revisaron 12 bases de datos bibliográficas electrónicas, citas relevantes en forma manual y se contactaron autores. Fueron incluidas 26 ensayos clínicos aleatorizados, 10 de ellos aleatorizados por clusters (clases, escuelas, clínicas o vecindarios) en lugar de individuos. Todos los estudios incluidos fueron realizados entre 1970 y 2000 en EE.UU, RU y Canadá, en su mayoría en poblaciones afroamericanas o hispánicas. Dos revisores evaluaron independientemente la calidad metodológica de los artículos originales.

\section{Resultados principales}

No hubo intervenciones efectivas para: 1) retrasar el inicio de las relaciones sexuales ni en mujeres ni en los varones jóvenes (ver cuadro) con consistencia de los resultados entre los diferentes estudios; 2) estimular en mujeres jóvenes el uso durante todas las relaciones sexuales de métodos para el control de la natalidad (el mismo efecto fue observado en los varones); 3) reducir la tasa de embarazos en mujeres jóvenes.

En cuatros estudios que evaluaron programas de abstinencia y en uno que evaluó un programa de educación sexual escolar se observó un aumento en el número de embarazos en las parejas de los varones jóvenes que participaron. Hubo una reducción significativa de embarazos en las mujeres que recibieron un programa multifacético.

\begin{tabular}{l|c|c}
\hline \multicolumn{2}{l|}{$\begin{array}{l}\text { Resultados } \\
\text { Inicio delas relaciones }\end{array}$} & OR (IC95\%) \\
sexuales & en mujeres jóvenes & $1,12(0,96-1,30)$ \\
\hline Control de lanatalidad & en varones jóvenes & $0,99(0,84-1,16)$ \\
\hline en las mujeres jóvenes & toda relación sexual & $0,95(0,69-1,30)$ \\
\hline Embarazos en las parejas de varones jóvenes & $1,05(0,50-2,19)$ \\
\hline Embarazos en mujeres en programas multifacéticos & $1,54(1,03-2,29)$ \\
\hline
\end{tabular}

\section{Conclusiones}

Las estrategias de prevención primaria evaluadas no retrasaron la iniciación de las relaciones sexuales, no mejoraron el control de la natalidad en mujeres y varones jóvenes ni redujeron el número de embarazos en las mujeres jóvenes.

Fuente de financiamiento: National Health Research Development Program, Health Canada; Ontario Ministry of Health and Long-Term Care; Region of

\section{Comentario}

Los autores advierten sobre algunas dificultades de los trabajos incluidos en esta revisión: 1) en todos los estudios menos uno el grupo control recibió una intervención convencional (programas vigentes en las escuelas o clínicas) en lugar de no intervención, lo que permitiría subestimar la eficacia de las intervenciones evaluadas por falta de potencia; 2) sólo ocho de los 22 estudios tuvieron un puntaje dos más puntos de los cuatro considerados en la evaluación de la calidad metodológica1 (sin embargo el pobre nivel metodológico tendería a sobreestimar los efectos del tratamiento en lugar de subestimarlos ${ }^{2}$ y es poco probable que pueda explicar el fracaso de las intervenciones); 3) la mayoría de las poblaciones incluidas en los estudios pertenecían a grupos de bajo nivel socioeconómico, lo que no descarta que estas intervenciones puedan ser exitosas en otras poblaciones; 4) no pudieron explicar la heterogeneidad entre los estudios que evaluaron el uso de métodos de control de la natalidad en mujeres jóvenes.

Debido a estas limitaciones los autores concluyen que la revisión muestra que aún no se tienen soluciones al problema de las altas tasas de embarazo adolescente en países como EEUU, RU y Canadá y sugieren investigar sobre los determinantes sociales del embarazo no planificado en adolescentes mediante estudios longitudinales que comiencen en etapas tempranas de la vida y utilizar estos resultados para planificar intervenciones preventivas. Estudiar los países con bajas tasas de embarazo adolescente sería importante al momento de pensar en estrategias que enseñen a los adolescentes lo que son las relaciones sexuales saludables.
Además, para mejorar la calidad de las relaciones sexuales entre adolescentes, éstos deberían participar en el diseño de las intervenciones.

Esta revisión incluyó estudios controlados con un buen diseño metodológico experimental que no mostraron beneficios de las intervenciones para mejorar las prácticas sexuales en adolescentes. Sería importante, también, hacer una revisión de estudios cuasiexperimentales* que no han sido incluidos en esta revisión. Los factores relacionados al éxito o fracaso de los programas ya realizados deben tenerse en cuenta e implementar estrategias que hayan resultado exitosas en otros países para evaluar su aplicabilidad en nuestro medio teniendo en cuenta las características propias de la población a la que asistimos, mediante estudios cualitativos que analicen los determinantes sociales del embarazo adolescente. Los programas de prevención requieren un fuerte componente de evaluación (sean experimentales o cuasi-experimentales) tanto del impacto como del proceso y quizás deban iniciarse más tempranamente como lo sugiere una revisión sistemática ${ }^{3}$.

Conclusiones del comentador: este estudio brinda importante información y es un llamado de atención al momento de planificar programas dirigidos a prevenir los embarazos no buscados en adolescentes.

Lic. María Belizán [ Instituto de Efectividad Clínica y Sanitaria (IECS), Buenos Aires, Argentina ]

Dra. María Luisa Cafferata [ Centro Latinoamericano de Perinatología y Desarrollo Humano (CLAP. OPS-OMS), Montevideo, Uruguay ]

Referencias:

1. Jadad AR, Moore RA, Carroll D, Jenkinson C, Reynolds DJM, Gavaghan DJ, et al. Assessing the quality of reports of randomized clinical trials: is blinding necessary? Control Clin Trials 1996; 17: 1-12

2. Guyatt GH, DiCenso A, Farewell V, Willan A, Griffith L. Randomized trials versus observational studies in adolescent pregnancy prevention. J Clin Epidemiol 2000; 53: 167-174 3. Zoritch B, Roberts I, Oakley A. Day care for preschool children. Cochrane Database Sys Rev 2000;(2):CD000564.

Palabras claves ${ }^{(5)}$

Embarazo adolescente, embarazo no deseado, control de la natalidad, programas de prevención, investigaciones clínicas aleatorizadas, revisión sistemática. 